\title{
Personality Traits for a Social Mediator Robot Encouraging Elderly Self-Disclosure on Loss Experiences
}

\author{
YOHEI NOGUCHI, University of Tsukuba \\ HIROKO KAMIDE, Nagoya University \\ FUMIHIDE TANAKA, University of Tsukuba
}

\begin{abstract}
To prevent elderly people from being socially isolated, encouraging their self-disclosure takes an important role. We discuss a use case of social robots in which they are deployed as mediators for humans that intermediate remote communication between elderly people and their family members or friends. The goal of this article is to present a design guideline for such social mediator robots based on results obtained from two studies in which a total of 741 elderly people participated. In study 1, we explored topics in dialogues and found that a social mediator robot could well encourage the self-disclosure of the elderly people, particularly in topics of which they usually feel resistance in talking to others (e.g., loss experiences). Thus, we confirmed the feasibility of the social mediator robot. Study 2 pursued the effective personality traits of the social mediator robot. We re-investigated a well-studied research question of matching robot personality to the user. The results provided more detailed knowledge as to similarity-attraction/repulsion than had been reported previously. Finally, design recommendations were discussed by considering the personality traits of the elderly users as well.
\end{abstract}

CCS Concepts: • Computer systems organization $\rightarrow$ Robotics; $\bullet$ Human-centered computing $\rightarrow$ Interaction design; User studies;

Additional Key Words and Phrases: Social mediator robot, self-disclosure, older persons, matching personality, robot design

\section{ACM Reference format:}

Yohei Noguchi, Hiroko Kamide, and Fumihide Tanaka. 2020. Personality Traits for a Social Mediator Robot Encouraging Elderly Self-Disclosure on Loss Experiences. Trans. Hum.-Robot Interact. 9, 3, Article 17 (May 2020), 24 pages.

https://oi.org/10.1145/3377342

\section{INTRODUCTION}

Social isolation in elderly people is becoming a serious issue in many countries. It is particularly the case with the elderly who live separately from other family members. In addition, it should be noted that elderly people often do not reveal their true emotions easily [11,36]. Even if they are

This work was supported by JSPS KAKENHI grants 15H01708 and 19H01112.

Authors' addresses: Y. Noguchi and F. Tanaka, University of Tsukuba, Tennodai 1-1-1, Tsukuba, Ibaraki, 305-8573, Japan; emails: noguchi@ftl.iit.tsukuba.ac.jp, fumihide.tanaka@gmail.com; H. Kamide, Nagoya University, Furo-cho, Chikusa-ku, Nagoya, Aichi, 464-8601, Japan; email: kamide@coi.nagoya-u.ac.jp.

Permission to make digital or hard copies of all or part of this work for personal or classroom use is granted without fee provided that copies are not made or distributed for profit or commercial advantage and that copies bear this notice and the full citation on the first page. Copyrights for components of this work owned by others than the author(s) must be honored. Abstracting with credit is permitted. To copy otherwise, or republish, to post on servers or to redistribute to lists, requires prior specific permission and/or a fee. Request permissions from permissions@acm.org.

(C) 2020 Copyright held by the owner/author(s). Publication rights licensed to ACM.

2573-9522/2020/05-ART17

https://doi.org/10.1145/3377342 


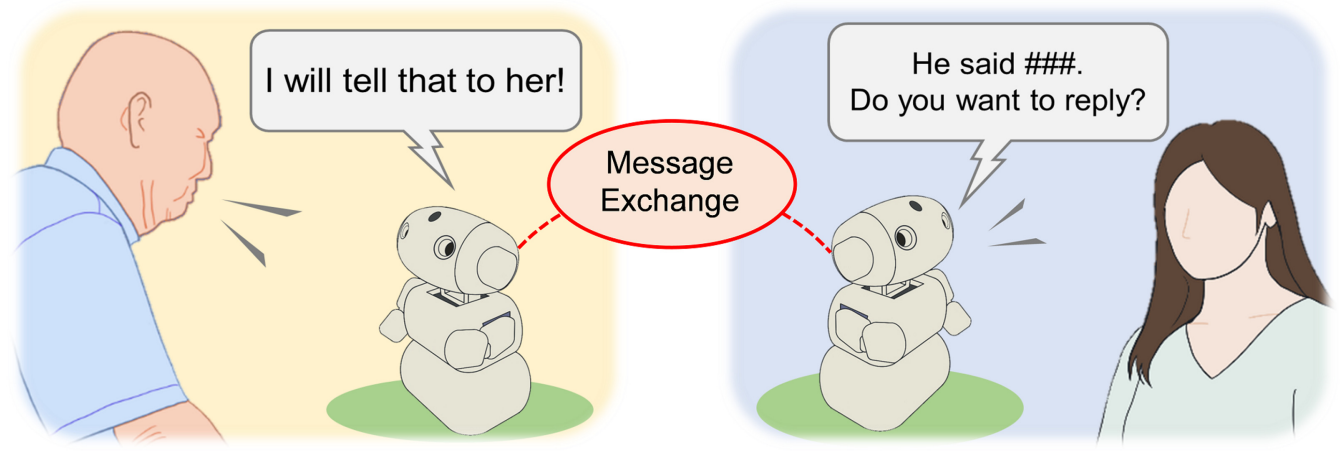

Fig. 1. Social mediator robots. Robots connected via the Internet serve as mediators, and humans can exchange their messages through the robots. In this study, we assume the elderly users who live separately from their family members or friends, and both sides, use the robots to exchange their messages.

facing serious troubles such as health or financial problems, it can be difficult for them to disclose the troubles to others and request supports. To prevent elderly people from being socially isolated, technological supports should provide them with a smooth communication capability with other people while encouraging their self-disclosure.

In this article, we will discuss social robots that serve as a remote communication interface between humans (Figure 1). In this framework, the social robots placed in each house are connected via the Internet, and humans can exchange their messages through the (mediator) robots. In such robot-mediated communication, several benefits are expected. Recent advancement in speech recognition technologies brings easier message creation than in the past. Although more improvements are needed, they are particularly welcomed by users who feel stress in key typing. Most importantly, if social robots mediate interpersonal communication [51,54] in an appropriate manner, self-disclosure of elderly people could be encouraged-for instance, it may become easier for elderly people to self-disclose in this way than directly talking over the phone. However, the concept of the social mediator robot, particularly in the context of encouraging elderly self-disclosure, is still unexplored, and little knowledge is available with respect to the design guidelines.

The goal of this article is to explore the design guideline of the social mediator robots that can help elderly users self-disclose and send their messages to remote recipients by performing large-scale exploratory studies. More specifically, using prototype robots developed based on preliminary surveys and workshops, we conducted two studies involving a total of 741 elderly participants. One of the studies was carried out in a laboratory setup in which 21 participants interacted with a mediator robot. The other study was performed based on online surveys involving a total of 720 participants by using video stimuli, exploring factors that affect elderly self-disclosure in using social mediator robots.

The two studies addressed two research questions illustrated in Figure 2. The results presented a design guideline for creating effective social mediator robots and contributed to the HRI researches concerning self-disclosure, elderly support, and remote communication. To summarize, the following knowledge was provided:

(1) Effective dialogue topics for social mediator robots (i.e., topics in which elderly people feel it is easier to self-disclose with the robots than by phones).

(2) Effective personality traits of social mediator robots according to the personality traits of the elderly user. 


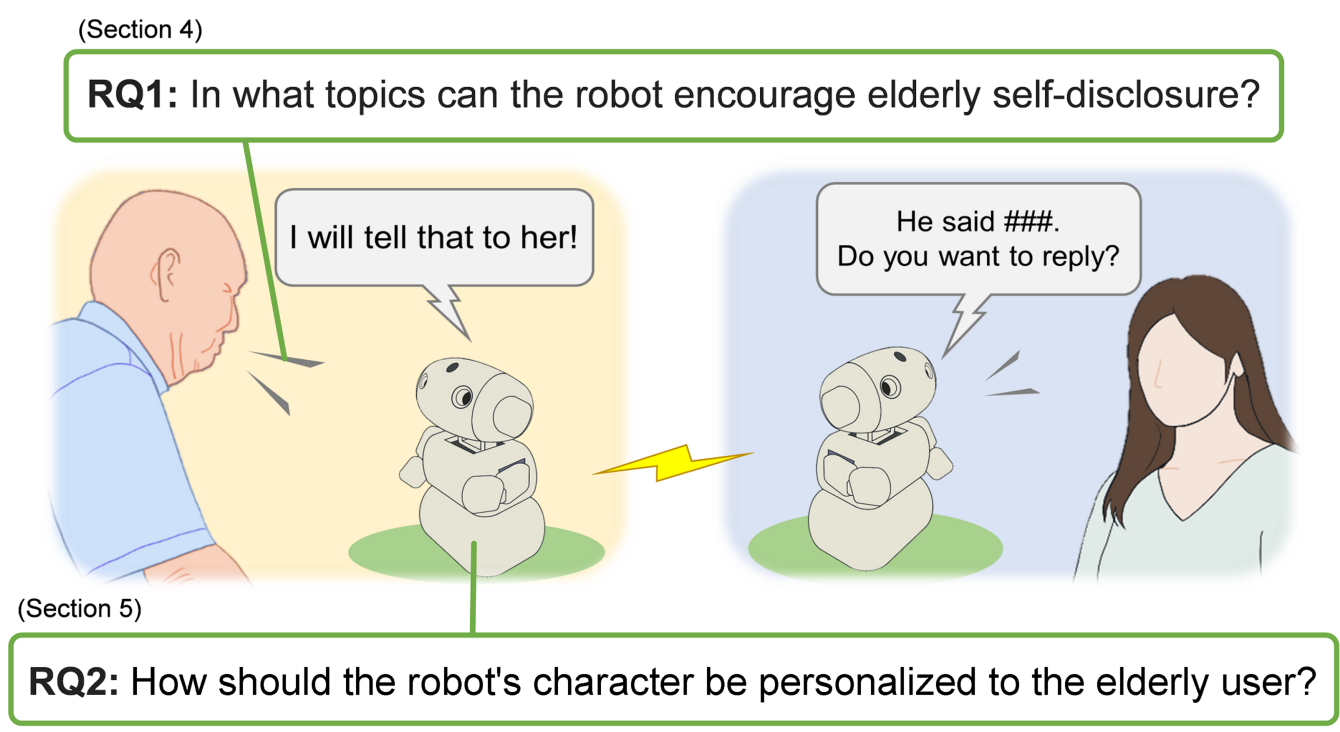

Fig. 2. Two research questions discussed in this article.

In the following sections, we first discuss related works (Section 2), explain prototype robots used in our studies (Section 3), and then describe two studies respectively in Sections 4 and 5. Section 6 summarizes general discussions and limitations of these studies, followed by our conclusion in Section 7.

\section{RELATED WORKS}

\subsection{Benefits of Self-Disclosure}

Self-disclosure has been of interest to philosophers, sociologists, and psychologists for a long time. In the Encyclopedia of Social Psychology, self-disclosure was referred to as "the process of revealing personal, intimate information about oneself to others" [7]. Jourard [27] pioneered a spectrum of research concerning human self-disclosure, including its relationship with a healthy personality. On the other hand, in developmental psychology, Erikson et al. [13, 14] defined the model of psychological conflict in elderly people as integrity vs. despair. Both are experienced when people reflect on their lives and think about what they have or have not accomplished. Self-disclosure becomes important in terms of integrity. To self-disclose life experiences from the viewpoint of integrity refers to sharing memories with people of the same generation and teaching morals to the younger generation; these act as therapeutic interventions for disclosers that mitigate their stress and enhance adaptation [21]. Furthermore, disclosing one's own troubles to other people can be a trigger for social support [56]. Social supports are classified into four aspects-emotional support, material support, informational support, and appraisal support [24]-and self-disclosure provides important information for these types of support.

Although there are many benefits in self-disclosure, troubles represented by loss experiences, such as loss of physical/mental health, loss of economic base, loss of social networks, and loss of reasons to live, are known as topics that are difficult for elderly people to disclose even to persons close to them (e.g., intimate friends or family members) [53]. However, interpersonal communication often becomes smooth if there is a third-person mediator. Therefore, the difficulty of self-disclosure could be alleviated, and elderly people might be encouraged to request social supports from others. 
Indeed, recent HRI research (see the next sections) suggests that, if designed appropriately, robots can elicit both self-disclosure and human-human interaction.

\subsection{Human-Robot Interaction and Self-Disclosure}

Relationships (e.g., trust, companionship, rapport) between disclosers and artificial entities have been regarded as a crucial factor of human self-disclosure [37, 57, 60]. In addition, there have been proposed interaction strategies for building relationships between the human and robot toward encouraging human self-disclosure (e.g., human-robot hugging [52], reciprocal self-disclosure [9], back-channeling [20]). These studies indicate that human self-disclosure can become deeper and broader as the relationship with the partner robot advances, based on the social penetration theory [1]. However, it was proposed that robots were regarded as social entities to which people could self-disclose easily because robots did not share social statements with humans [58]. It was also reported that, compared with communication between humans, human self-disclosure of negative topics tended to be encouraged when humans communicated with a visually simple robot [31]. Overall, these studies suggest that personal robots can encourage the self-disclosure of negative topics, such as loss experiences, in elderly people.

\subsection{Robot-Mediated Communication}

In the context of robot-mediated communication, telepresence robots and social mediator robots that are used in real-time human-human communication have been discussed. For remote communication support, telepresence robots have been used as mediators of interpersonal communication, and the applicability was tested for elderly support (e.g., health care [8, 30], working support $[23,44])$. Furthermore, social mediator robots that support local interpersonal communication were also proposed $[47,51,54]$. These studies suggest that through interaction with mediator robots, humans may be encouraged to communicate with other people (e.g., elderly people are motivated to interact with caregivers during their interaction with a Paro robot [51]).

Although these studies show the potential of mediator robots for elderly users, design knowledge with respect to encouraging self-disclosure remains insufficient. Messaging functions in robots have been studied (e.g., [29, 50]), but no effects have been reported on the self-disclosure of elderly people, and effective design guidelines for social mediator robots have not been provided. Furthermore, individual differences become a particularly important element when using social robots in mental health care [46], and thus mediator robots should be designed in accordance with individual user properties (e.g., personality traits, gender). It is considered that mediator robots should be able to adapt to each user depending on dialogue topics and the personality traits of the user.

\section{PROTOTYPE ROBOTS USED IN THIS STUDY}

\subsection{General Features}

Figure 3 shows the appearance of two robots that were used in our studies explained in the following sections. The robots had been regarded as test beds (not final products) for our studies to explore important factors for designing mediator robots. Therefore, each of the robots was designed in a simple appearance to minimize the influence of robot appearance and prior knowledge on commercial robots. Based on opinions obtained at pilot workshops involving elderly persons who had been participating in our research projects as leaders for elderly participants, these robots were designed in a size to be used on a tabletop. In addition, following the knowledge reported in Luria et al. [35], two eyes were placed to make the robots for home use.

Arduino microcontroller boards were used to control the servos. They were connected to laptops or small CPU boards (we tested on Raspberry Pi and Latte Panda) with Python scripts running. 


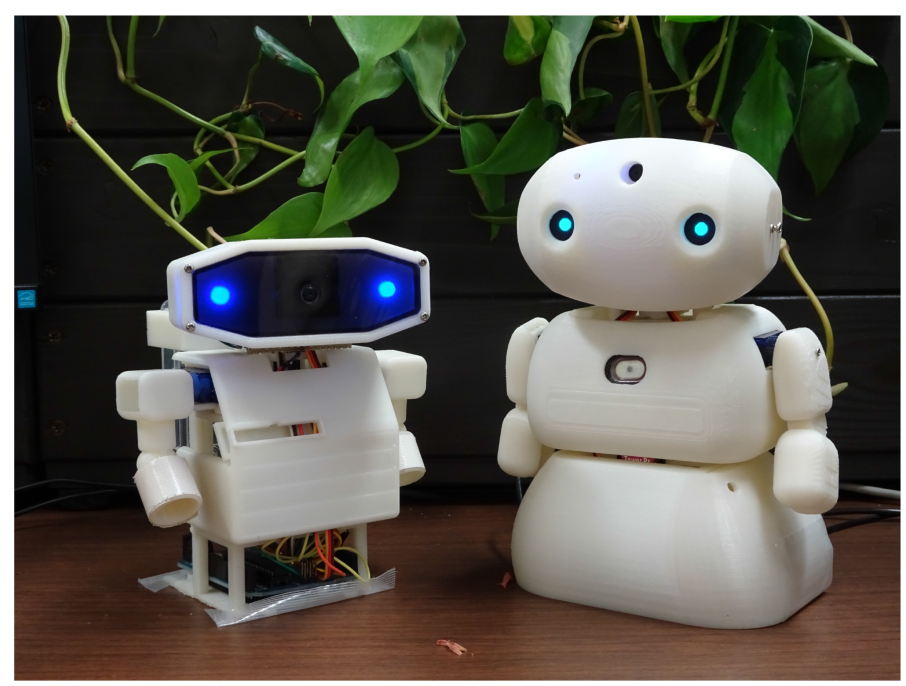

Fig. 3. Robots used in our studies.

The Python scripts provided speech, web server access, and high-level robot control, whereas the Arduino controlled the hardware that provided the physical expression. For the voice interaction function, we used the Google speech-to-text API for speech recognition and a text-to-speech API provided by NTT Docomo for speech synthesis. We developed an https web server to manage messages that were posted and requested from the robots.

\subsection{Type-A Robot}

The Type-A robot $(145 \mathrm{~mm}(\mathrm{H}) \times 130 \mathrm{~mm}(\mathrm{~W}) \times 105 \mathrm{~mm}(\mathrm{D}))$ is shown in the left side of Figure 3 . It is the first prototype we developed based on the feedback obtained from a pilot study [43]. It has 4 degrees of freedom (DOFs) (2 DOFs on the neck; head nod and swing, and 2 DOFs on the arms; left and right). The eyes are represented by two RGB LEDs. A microphone can be mounted on its right/left hand, and a camera is embedded on the face. This robot does not have a speaker module; thus, an external speaker is required. The Type-A robot was used in study 1 (Section 4).

\subsection{Type-B Robot}

The Type-B robot $(185 \mathrm{~mm}(\mathrm{H}) \times 155 \mathrm{~mm}(\mathrm{~W}) \times 130 \mathrm{~mm}(\mathrm{D}))$ is shown in the right side of Figure 3 . To conduct study 2 (Section 5), more DOFs in the head part were required than the case with the Type-A robot, and therefore we developed this Type-B robot. It has 6 DOFs (3 DOFs on the neck; head nod, tilt, and swing, 2 DOFs on the arms; left and right, and 1 DOF for body rotation). The eyes are represented by micro OLED displays (SparkFun LCD-13003) and controlled by Arduino Nano using the SPI communication method. A camera with a microphone module is built into the head, and a small speaker module is mounted on the chest.

\section{STUDY 1: TOPICS IN WHICH SELF-DISCLOSURE IS ENCOURAGED}

The goal of study 1 is to explore the feasibility of a social mediator robot for elderly people while addressing the first research question, RQ1, raised in Figure 2, namely about topics in which elderly self-disclosure is encouraged by the introduction of mediator robots. To this end, we carried out an HRI experiment in a laboratory setup and investigated the topics. We also analyzed a relationship 
between participants' social perception toward the mediator robots with different behaviors and the ease of self-disclosure.

\subsection{Hypothesis}

As reported in the area of computer-mediated communication (CMC), self-disclosure of humans tends to be encouraged [26,39], particularly under a condition in which the effect of non-verbal cues is small [59]. A similar effect could be expected with mediator robots because the effect of non-verbal cues of humans exchanged via the robots can be controlled. In addition, expressive behaviors of social robots encourage self-disclosure [32,37], and social behaviors such as greetings, nodding, and blinks are more acceptable for elderly people [22]. When topics are not so intimate for individuals (e.g., everyday experiences or their own positive memories), it is easy for them to disclose regardless of communication media. However, mediator robots equipped with social expressive behaviors could affect elderly self-disclosure even on intimate topics such as loss experiences. Therefore, we constructed the following hypothesis:

H1. In topics concerning loss experience, elderly self-disclosure is encouraged when a mediator robot having socially expressive behaviors is used.

\subsection{Participants}

In conducting an experiment, a communication scenario between an elderly "parent" $(N=21)$ and a "daughter" was prepared. The reasons for using this scenario were as follows: (1) as described initially in Section 1, we ultimately aimed for tackling the issue of social isolation in elderly people who lived separately from their family members, and (2) a study suggested that daughters tended to communicate with their parents more frequently than sons [49]. With this in mind, we selected elderly participants who actually had adult daughters. The average age of the parent participants was 72.0 ( $S D=5.12$; 9 men, 12 women). A female college student who often communicated with her parents took the role of the daughter, being a recipient of self-disclosure. The details of the scenario used in the experiment are described in Section 4.6. This study was conducted with the approval of the research ethics committee of the faculty of engineering, information, and systems of the University of Tsukuba (2015R109-3), and consent was obtained from each participant based on an informed concept.

\subsection{Experimental Design}

The medium of communication used by the participants was manipulated in the experiment. There were three conditions: (1) phone condition, in which phones were used but no robot, (2) expressive robot condition, in which a mediator robot with social expressions was used, and (3) nonexpressive robot condition, in which a mediator robot with no social expression was used.

When using the robot in (2) and (3), messages were converted from voice to text and sent to the daughter's smartphone remotely (the robot was used on the elderly person's side only). The experiment was designed to be accompanied by vocalization under all conditions to avoid creating a gap of cognitive load on task. Every participant joined in on all three conditions in a random order. For measuring the ease of self-disclosure, only the data obtained from the first condition for each participant were used (between-participants factor) to control the degree of their experience with the robot. However, for measuring social attributes toward the robot, data were collected and analyzed for a within-participant factor.

\subsection{Robots Used in the Experiment}

The Type-A robot (on the left in Figure 3) was used as a base platform. Then, we programmed in two different ways, with the following two robots used in the experiment. 
4.4.1 Expressive Robot. This robot responded to two types of voice commands, "send a message" and "check a message." It was also equipped with verbal and non-verbal expressions. When participants called "send a message," the robot nodded and then said "What should I tell her?" Recording began with a microphone mounted on the right arm of the robot. The recording continued for 20 seconds, and afterward the robot asked for confirmation and sent it to the daughter. Alternatively, when participants called "check a message," the robot nodded and then said "I will check messages, please wait a moment." Then, if there was any message, the robot read it out loud. If there was no message, the robot just said "I do not have any new message."

4.4.2 Non-Expressive Robot. In contrast, the robot for the non-expressive condition used beeps and LED colors. The robot responded to the same two voice commands as the case with the expressive robot. When the "send a message" command was called, the robot sounded a 587-Hz beep and began recording the user's voice for 20 seconds. Then, a $494-\mathrm{Hz}$ beep sounded to notify the end of the recording, and the voice message was sent to the daughter's smartphone. When the "check a message" command was called, the robot read the message out loud if any new message arrived. If not, the robot beeped at $494-\mathrm{Hz}$ twice.

\subsection{Measurements}

4.5.1 Ease of Self-Disclosure. The self-disclosure scale created by Suganuma [53] was used to evaluate the ease of self-disclosure under each condition. This scale comprises 16 items and three factors: everyday experience (4 items), integrated life experience (7 items), and loss experience (5 items). By using a questionnaire, the ease of self-disclosure can be measured on a scale from " 1 : quite hard to talk" to "6: quite easy to talk."

4.5.2 Social Attribute of Robots. The Robotic Social Attributes Scale (RoSAS) [10] was used. This scale has six items on each of the three factors: warmth, competence, and discomfort, and it was reported that robots with human-like appearance scored higher warmth and competence than robots with machine-like or human-machine blended appearance. In our study, the scale was used for a manipulation check to confirm that warmth and competence judgments on a mediator robot with social expression were greater than a robot with mechanical expression. This is because the social expression of robots was known to enhance people's perception of robots as more human like [17].

4.5.3 Impression on the Recipient. Because participants' subjective impressions to the recipient (daughter) were considered to affect the ease of self-disclosure, we inquired impressions on the daughter person of the participants, based on a scale of " 1 : She was hard to speak with" to " 5 : She was very easy to speak with.” The data were used as a covariance in later analyses (Section 4.7.2).

\subsection{Experimental Procedure}

Each elderly participant was given a general explanation about this study and was asked for the participant's consent at the beginning of the study. In consequence, all participants consented to all terms, including the disclosure of their personal information. Then, the elderly participant had a short meeting with a daughter person, in which they were introduced to each other. After that, both of them left and went into two separate rooms. Figure 4 shows one of the rooms for the elderly participants. At this moment, the elderly participant was provided with a scenario for the experiment, in which he or she was supposed to speak of a personal episode based on three subjects: recent enjoyment, an unforgettable experience, and health concerns. These were selected from a questionnaire used to measure the ease of self-disclosure described in Section 4.5.1. The 


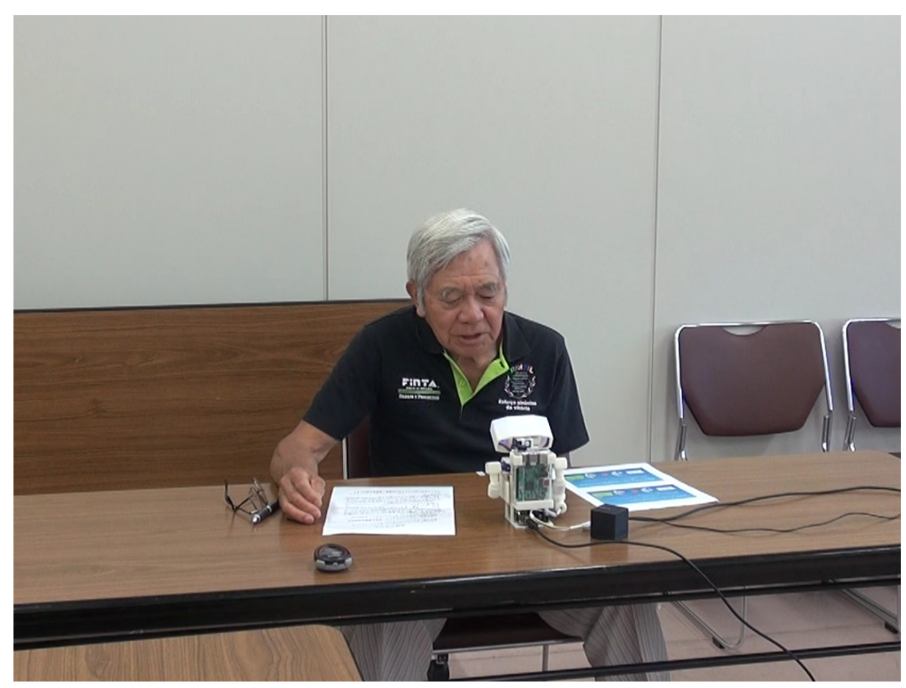

Fig. 4. A participant speaking to a mediator robot.

elderly participant was given a paper and was instructed to write the three episodes on the paper in advance so that he or she would be able to explain each episode in 20 seconds.

The daughter person was instructed and trained in advance such that she was supposed to receive a call/message from her parent who lived separately from her. In responding to each call/message, she was allowed to use only three kinds of replies: for recent enjoyment topics, "That's funny! Good for you!"; for unforgettable experience topics, "Did that really happen? It's unforgettable"; and for health concerns topics, "That must be concerning for you. We need to be careful about health."

In the phone condition, the elderly participant was given a smartphone and was instructed to call when he or she was ready. Upon connecting with the daughter person, and after initial greetings, the participants discussed the three topics.

In the expressive robot and non-expressive robot conditions, there was a training phase at first in which each participant could get used to robot-mediated communication by being instructed how to use the robot, how to send messages, and how replies from the daughter person would be received. After the training phase, the participant was guided into the communication task with the daughter person.

During the experiment, the experimenter was waiting outside the room. On average, it took about 3 minutes per session for the phone condition and about 10 minutes per session for the robot conditions including the transaction time. After the communication task ended, the participant was asked to answer the questionnaire described in the previous section.

\subsection{Results}

4.7.1 Manipulation Check. For a warmth factor in RoSAS, the expressive robot was rated significantly warmer $(M=4.32, S D=.90)$ than the non-expressive robot $(M=3.67, S D=1.06$; $t(20)=3.02, p=.007, d=.66$ ). For a competence factor, the expressive robot was rated significantly more competent $(M=4.44, S D=1.04)$ than the non-expressive $\operatorname{robot}(M=3.76, S D=1.00$; $t(20)=3.05, p=.006, d=.67)$. From these results, the robot with social expression was perceived as more expressive. Therefore, we concluded that the behaviors of the robots were well manipulated. 


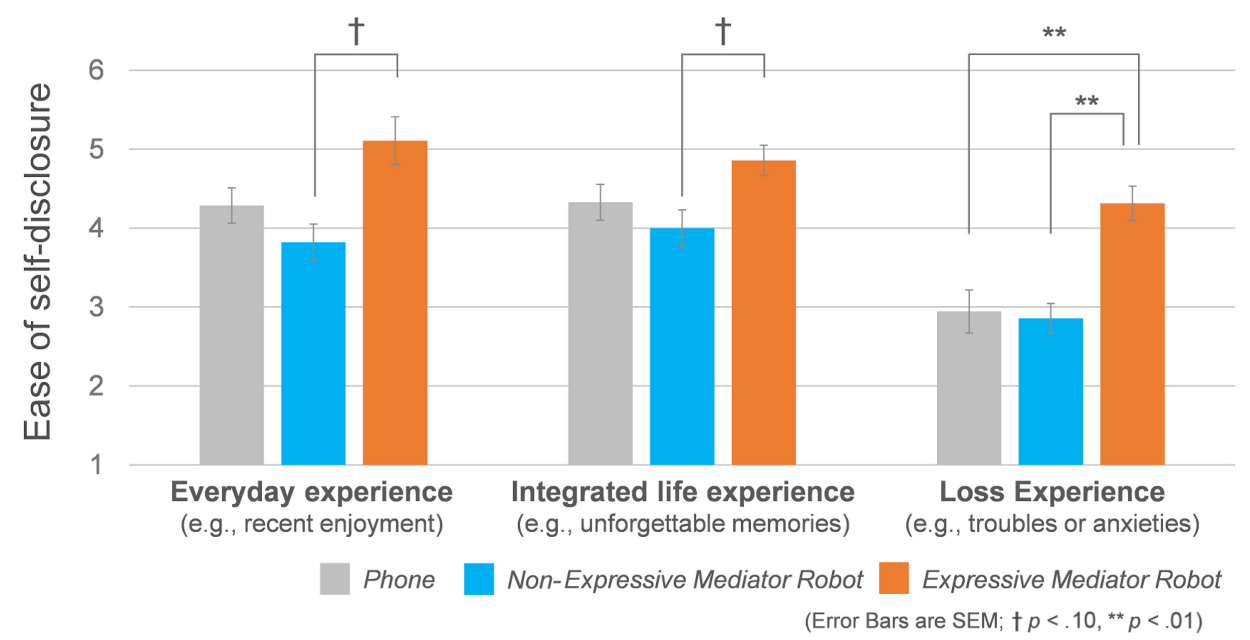

Fig. 5. Ease of self-disclosure through different communication media. The results show that elderly participants judged the mediator robot with social expressions (expressive robot condition) as the easiest medium for self-disclosing their loss experiences.

4.7.2 Statistical Analysis. First, we examined if participants' impression on the daughter person (ease of talking with) affected their ease of self-disclosure. To this end, we performed a parameter estimation by a linear regression analysis with the ease of self-disclosure as a dependent variable, the communication medium as an independent variable, and the impression on the daughter person as a covariance. Then, in topics concerning everyday experience, the effect of the covariance was found to be significant $\left(B=.63, F(1,17)=15.1, p=.001, \eta_{p}{ }^{2}=.47\right)$. In contrast, no significant coefficient of the covariance was found in topics concerning integrated life experience $\left(B=.24, F(1,17)=1.74, p=.20, \eta_{p}{ }^{2}=.093\right)$ nor in topics concerning loss experience $(B=.28$, $\left.F(1,17)=2.28, p=.15, \eta_{p}^{2}=.12\right)$. Furthermore, a two-way ANOVA showed no significant interaction between the communication medium and the impression on the daughter person in topics concerning everyday experience $\left(F(2,15)=1.51, p=.25, \eta_{p}^{2}=.17\right)$. Thus, it was concluded that the impression of the daughter person affected the ease of self-disclosure only in topics concerning everyday experience, which was independent of the communication medium.

Therefore, in examining the effect of a communication medium factor on the ease of selfdisclosure, we decided to use a one-way ANCOVA with the impression on the daughter person as a covariance in topics concerning everyday experience and use a one-way ANOVA in other topics.

4.7.3 Ease of Self-Disclosure. Figure 5 shows the average scores of the ease of self-disclosure of each communication medium of three kinds of topics.

As explained in the previous section, for topics concerning everyday experience, a one-way ANCOVA was performed. The results showed a marginal effect of the medium factor $(F(2,17)=2.96$, $\left.p=.079, \eta_{p}{ }^{2}=.26\right)$. Post hoc comparisons using $t$-tests with Bonferroni correction showed that there was a marginally significant difference between the expressive robot condition $(M=5.11$, $S D=.86)$ and the non-expressive robot condition $(M=3.82, S D=.66 ; t(17)=2.33, p=.097$, $d=1.31)$. No significant difference was found between the phone condition $(M=4.29, S D=.64)$ and the other two robot conditions.

Then, for topics concerning integrated life experience and loss experience, a one-way ANOVA was performed. In topics concerning integrated life experience, there was a marginally significant effect of the medium factor $\left(F(2,18)=3.40, p=.056, \eta_{p}^{2}=.27\right)$. Post hoc comparisons showed 
that the mean score for the expressive robot condition $(M=4.86, S D=.55)$ was marginally larger than the case with the non-expressive robot condition $(M=4.00, S D=.66 ; t(18)=2.58, p=.056$, $d=1.41)$. No significant difference was found between the phone condition $(M=4.33, S D=.65)$ and the other two robot conditions.

Finally, in topics concerning loss experience, there was a significant effect of the medium factor $\left(F(2,18)=10.9, p=.001, \eta_{p}{ }^{2}=.55\right)$. Post hoc comparisons showed that the mean score for the expressive robot condition $(M=4.31, S D=.62)$ was significantly larger than the cases with the phone condition $(M=2.94, S D=.78 ; t(18)=3.92, p=.003, d=1.95)$ and the non-expressive robot condition $(M=2.86, S D=.54 ; t(18)=4.17, p=.002, d=2.51)$.

\subsection{Discussions and Limitations}

The results suggest that the mediator robot with social expressions was regarded as more expressive, and when elderly participants self-disclosed through such a medium, the ease of selfdisclosure increased, particularly when talking about their loss experiences. Therefore, hypothesis H1 was considered to be supported.

In telecommunication using the mediator robot, the effect of non-verbal cues transmitted in real time was much smaller than that of phone or video calls. It was reported in CMC research that communication media with weak non-verbal cues (e.g., emails) positively affect human selfdisclosure due to small cognitive loads and selective self-presentation [59]. However, no significant difference was observed between the non-expressive robot condition and the phone condition. This might have been because the robot required the speech of the users, which was different from previous $\mathrm{CMC}$ devices using keyboard inputs. Users tend to hesitate and feel embarrassed if the response of a robot is not good. Mediator robots should be designed so that their social expressions in making responses can be perceived as expressive.

It is noteworthy that the perception of a mediator robot as expressive could affect elderly selfdisclosure much stronger than its functional specification, such as the number of non-verbal cues or asynchronicity. The results also show that both the verbal and non-verbal expressions, implemented in the expressive robot condition, are examples of behaviors that strengthen human perception of the mediator robot as a social entity.

However, we also have to be aware of the limitations of this study. First, the sample size $(N=7$ per condition) of study 1 was small. Next, there were multiple factors between the phone condition and the robot conditions, and the study did not completely control them. For example, in the phone condition, participants spoke in real time with the confederate, although the conversation was controlled to some extent by a pre-defined script and response patterns, whereas in the robot conditions, the conversation was strictly made step by step.

In conclusion, study 1 demonstrated the feasibility of the social mediator robot to encourage the self-disclosure of elderly speakers, particularly in dialogue topics concerning loss experiences.

\section{STUDY 2: EFFECTIVE PERSONALITY TRAITS FOR MEDIATOR ROBOTS}

Study 2 explores the second research question, "RQ2: How should the robot's character be personalized to the elderly user?" illustrated in Figure 2. To this end, we conducted an online survey research in which 720 elderly people participated. Video stimuli, each of which contained a type of mediator robot with one of multiple personality traits, were used in the survey.

\subsection{Hypotheses}

In the HRI research field, it is well known that matching robot personality brings a positive influence on the humans' task performance [2, 5, 33, 38, 55]. The extroversion dimension of the Big Five personality model [25] was often employed, and the theory of similarity-attraction-that is, 
a person was attracted more to others who were matched in personality than to those who were mismatched-was discussed [41,42]. It was also reported that mutual self-disclosure increased among similar extroverted dyads [12]; therefore, a match in extroversion between a robot and the user could positively affect the depth of the user's self-disclosure.

However, the amount of self-disclosure tends to become less from elderly people with lower self-esteem [53]. Self-esteem concerns the adaptation levels of humans [28] and is known to be negatively correlated with neuroticism [4], which is another dimension of the Big Five personality model. Therefore, in a case with high neuroticism (low self-esteem), even if the personality of a robot is matched to the one of the user, the user's self-disclosure to the robot could be suppressed. As a consequence, we have the following hypotheses:

H2a. Matching personality traits of a mediator robot to the elderly user with low neuroticism increases his/her depth of self-disclosure (similarity-attraction).

H2b. Matching personality traits of a mediator robot to the elderly user with high neuroticism decreases his/her depth of self-disclosure (similarity-repulsion).

To distinguish similarity-attraction and similarity-repulsion statistically, we need to consider at least one more personality trait (variable) other than neuroticism. Therefore, in this study, we will observe not only main effects concerning neuroticism but also interactions involving extroversion, then discuss both the similarity-attraction and similarity-repulsion, whose definitions are as follows:

Similarity-attraction: The depth of self-disclosure of an elderly user increases if personality traits (neuroticism and extroversion) of a mediator robot match the ones of the elderly user compared with the cases with a single match (matched in either neuroticism or extroversion).

Similarity-repulsion: The depth of self-disclosure of an elderly user decreases if personality traits (neuroticism and extroversion) of a mediator robot match the ones of the elderly user compared with the cases with a single match (matched in either neuroticism or extroversion).

\subsection{Participants}

Participants for this study were recruited through MACROMILL Inc., which is a leading market research company in Japan. Initially, we obtained 720 participants older than 65 years (all Japanese, $M=69.8$ years, $S D=4.11,50 \%$ female and $50 \%$ male). However, we excluded data obtained from 131 participants who were not confident in understanding all of the survey procedure (measured by a question item), and therefore data from 589 participants were used in analyses in the end. The target profiles of participants in the recruitment were (1) persons who were communicating with their own children and intimate friends at least once in 3 years by phone or face-to-face, (2) persons who were living alone or with their spouses only, (3) persons who had own troubles regarding either of the loss experience topics of health, financial, isolation, and reasons to live ${ }^{1}$ that had never been disclosed to their family members or friends. To exclude participants who did not meet those requirements, a screening process was carried out before proceeding to the main survey. The fieldwork period was from December 17 to December 19 of 2018 and was conducted by MACROMILL Inc.

\footnotetext{
${ }^{1}$ In the questionnaire, we asked: "Do you have experiences regarding troubles concerning your own health and illness (health), troubles concerning living income and savings (finance), troubles concerning people whom you can depend on disappearing and you become alone (isolation), or you cannot find reasons to live (reasons to live)?"
} 
Table 1. Examples of Simulated Human-Robot Conversations

(a) HE+HN Robot

\begin{tabular}{ll}
\hline Speaker & \\
\hline \hline Discloser: & "I want to send a message.” \\
Robot: & That's what I have been waiting for. \\
& You can send a message to that person! Please say anything! \\
Discloser: & "Recently, I'm in trouble with **, and I'd like you to listen to me...” \\
Robot: & Ok, I see... That must have been really hard for you. \\
& I will pass your message responsibly. \\
& But I know you have such a problem, I'm in panic right now. \\
& I'm feeling awkward.
\end{tabular}

(b) LE+LN Robot

\begin{tabular}{|c|c|}
\hline Speaker & Utterance \\
\hline Discloser: & "I want to send a message." \\
\hline Robot: & OK. You can send a message to that person. So, what is your message? \\
\hline Discloser: & "Recently, I'm in trouble with **, and I'd like you to listen to me..." \\
\hline \multirow[t]{3}{*}{ Robot: } & Ok, I see... That must have been really hard for you. \\
\hline & I will pass your message responsibly. \\
\hline & It is important to calm down at such a moment. I will handle myself well. \\
\hline
\end{tabular}

Note: In both of these examples, the first half concerns the extroversion trait (HE/LE) of the robot, and the second half concerns the neuroticism trait $(\mathrm{HN} / \mathrm{LN})$ of the robot.

\subsection{Video Stimuli}

Pre-recorded video clips simulating human-robot conversations taken from the first-person perspective were used. There were four video clips, each of which contained one of four robots with different personality traits (high/low extroversion and high/low neuroticism). The robots were based on a Type-B robot (on the right side in Figure 3). The time length of each video clip was about 40 seconds. The dialogues taken in the video clips consisted of two parts: in the first half, the robot behaved to express its extroversion, whereas neuroticism was expressed through its behaviors in the second half. Examples of the simulated conversations are shown in Table 1. Although the voices of the robots were recorded, the utterances of disclosers were presented by subtitles only (no voice). These video clips are available from the links in the footnote. ${ }^{2}$

Studies in psychology showed that extroverts tend to have a higher speech rate and produce more utterances and also have higher gesture rates than introverts [19, 34]. These traits have also been tested in artificial agents $[2,5,33,38,55]$. Following this knowledge, we implemented extroverted and introverted behaviors with verbal (voice pitch, speed, and phrases) and non-verbal (the speed/frequency of head nodding, tilt, gaze, and arm movements) expressions. For vocal expressions, we used a text-to-speech API provided by NTT Docomo. The high extroversion (HE) robot spoke more phrases than the low extroversion (LE) robot, expressing joy with $+10 \%$ pitch and $+10 \%$ speed, together with configuring an emotion parameter provided by the API with "happiness." In contrast, the LE robot spoke fewer phrases than the HE robot, in neutral pitch and speed, configuring the emotion parameter with "neutral." The HE robot held eye contact, whereas the LE robot was programmed with a gaze aversion based on a model reported in Andrist et al. [6],

\footnotetext{
${ }^{2} \mathrm{HE}+\mathrm{HN}$ robot: https://youtu.be/z-f6Gk4-XbY; HE+LN robot: https://youtu.be/IYP9q9kN-z4; LE+HN robot: https://youtu. be/2EnGWRNYE-0; LE+LN robot: https://youtu.be/E-4Pasn4EYE.
} 
looking to its top, left, right, and bottom once every 7 seconds, by a ratio of 1:2:2:5. The HE robot also showed head and arm movements two times faster and more frequently than the LE robot.

It was reported that people with high neuroticism are looked at as being forced, awkward, or strained by their interlocutors during interactions $[12,18]$. We then implemented the high neuroticism $(\mathrm{HN})$ robot such that it showed signs of tension or anxiety during interactions. The HN robot verbally expressed insecurity and sensitivity by $+10 \%$ pitch and $+10 \%$ speed, ${ }^{3}$ together with configuring the emotion parameter with "sadness." In addition, to show awkwardness, the robot trembled several times with its head and arm. However, the low neuroticism (LN) robot verbally expressed emotional stability toward the user's self-disclosure in a stable speed and pitch.

\subsection{Measurements}

5.4.1 Perception of Robot Personality Traits. For the purpose of a manipulation check, we measured how participants perceived different personality traits of the robots in the video stimuli explained in the previous section. The Japanese version of the Ten-Item Personality Inventory (TIPI-J [45]) was used to evaluate the extroversion and neuroticism of the robots. Question items for evaluating the robot's extroversion were "This robot is extraverted and/or enthusiastic" and "(-) This robot is reserved and/or quiet," and question items for evaluating the robot's neuroticism were "This robot is anxious and/or easily upset" and "(-) This robot is calm and/or emotionally stable." A 7-point scale of "1: strongly disagree" to "7: strongly agree" was used in answering these question items.

5.4.2 Depth of Self-Disclosure to the Robot. To measure the depth of the participants' selfdisclosure to the robot, we asked them to evaluate how much they wanted to talk to the robot. A 7-point scale of "1: I do not want to talk at all" to "7: I want to talk in full details" was used for this measurement.

5.4.3 Personality Traits of Participants. A Big Five scale [40] was used to evaluate the extroversion and neuroticism of each participant, which was represented by five items: extroversion: $(-)$ quiet, sociable, talkative, extrovert, and cheerful; neuroticism: uneasy, anxious, discouraged, nervous, and depressive. A 7-point scale of "1: strongly disagree" to "7: strongly agree" was used in answering the question items.

5.4.4 Self-Esteem in Participants. Rosenberg's self-esteem scale [48] was used to check correlations between participants' self-esteem and neuroticism. A 4-point scale consisting of "1: strongly disagree," "2: disagree," "3: agree," and "4: strongly agree" was used for this survey.

\subsection{Procedure}

At the beginning of the study, an experimenter explained the basic concept of mediator robots and the purpose of this study to each participant. Then, the participant was instructed to watch four video clips explained in Section 5.3 in an assigned random order. After that, the participant was asked to answer questions about robot personality (Section 5.4.1) and self-disclosure (Section 5.4.2), followed by another set of questions as to the participant's personality (Section 5.4.3) and selfesteem (Section 5.4.4). Last, as explained in Section 5.2, the participant's comprehension about this survey was checked by using the two questions: "I could not understand some video contents" and "I could not understand how the mediator robot works." The participant could assess his or her comprehension based on a 7-point scale of "1: strongly no" to "7: strongly yes." Results data obtained from the participants who marked from a 5 to a 7 on either or both of the questions were excluded from analyses.

\footnotetext{
${ }^{3}$ In the case of the HE+HN robot, the pitch and speed were both increased a total of $20 \%$ from their baselines.
} 
Table 2. Participant Structure Divided by Personality Traits and Topics Chosen by Them at the Time of Their Recruitment

Personality Traits

\begin{tabular}{|c|c|c|c|c|}
\hline Topics & $\mathrm{HE}+\mathrm{HN}$ & $\mathrm{HE}+\mathrm{LN}$ & $\mathrm{LE}+\mathrm{HN}$ & $\mathrm{LE}+\mathrm{LN}$ \\
\hline$\overline{\text { Health }}$ & 33 (18 males) & "56 (22 males) & "24 (15 males) & 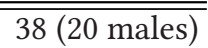 \\
\hline Finance & 19 (7 males) & 45 (22 males) & 38 (13 males) & 37 (23 males) \\
\hline Isolation & 27 (14 males) & 41 (18 males) & 48 (26 males) & 35 (20 males) \\
\hline Reasons to live & 32 (11 males) & 29 (14 males) & 53 (31 males) & 34 (18 males) \\
\hline Total $(N=589)$ & 111 (50 males) & 171 (76 males) & 163 (85 males) & 144 (81 males) \\
\hline
\end{tabular}

HE, high extroversion.; LN, low neuroticism.

\subsection{Experimental Design and Statistical Analysis}

Participants were divided into $2 \times 2$ groups based on high/low extroversion and high/low neuroticism by using median values ${ }^{4}$ obtained from the Big Five scale (Section 5.4.3) with a boundary value of 4.00. The structure of the participants is summarized in Table 2.

On all topics and personality traits, a $2 \times 2 \times 2$ split-plot factorial ANOVA was performed on the depth of self-disclosure to each robot, with the factors of the gender of participants (betweenparticipants factor), extroversion traits of the robot (within-participants factor), and neuroticism traits of the robot (within-participants factor). ${ }^{5}$

\subsection{Results}

5.7.1 Manipulation Check: Perception of Robot Personality Traits. Before using repeated measure one-way ANOVA, we performed Mauchly's sphericity test, which showed that the violation of sphericity occurred on each personality trait (extroversion: $\chi^{2}(2)=56.0, p<.001$; neuroticism: $\left.\chi^{2}(2)=213.8, p<.001\right)$. Therefore, we corrected the DOFs by using Greenhouse-Geisser estimates of sphericity (extroversion: $\epsilon=0.94$; neuroticism: $\epsilon=0.80$ ). Then, repeated measure one-way ANOVA was performed. The results showed significant differences in participants' perception of the robot's extroversion $\left(F(2.83,1665)=104.6, p<.001, \eta_{p}{ }^{2}=.15\right)$ and neuroticism $\left(F(2.40,1411)=254.7, p<.001, \eta_{p}{ }^{2}=.30\right)$, and $t$-tests with Bonferroni correction showed that the personality traits of the robots were manipulated successfully: the robots with high extroversion were perceived higher in extroversion traits than the robots with low extroversion $(p<.001){ }^{6}$ and the robots with high neuroticism were perceived higher in neuroticism traits than the robots with low neuroticism $(p<.001){ }^{7}$

5.7.2 $H E+H N$ Participants. The marginal means of the depth of self-disclosure obtained from the $\mathrm{HE}+\mathrm{HN}$ participants are shown in Figure 6(a). There was a significant main effect of the neuroticism trait of the robots in two kinds of topics: the depth of self-disclosure to LN robots

\footnotetext{
${ }^{4}$ The mean extroversion value was $4.95(S D=.60)$ in the HE group and $3.22(S D=.66)$ in the LE group. There was a significant difference between them $(t(587)=32.9, p<.001, d=2.72)$. The mean neuroticism value was $4.84(S D=.56)$ in the HN group and $3.28(S D=.62)$ in the LN group. There was a significant difference between them $(t(586.0)=32.3$, $p<.001, d=2.66$; the assumption of homoscedasticity was violated.)

${ }^{5}$ In this study, we first divided participants into four groups based on their personality traits (extroversion and neuroticism) and then performed ANOVA in each of those groups. Therefore, our ANOVA did not include the factors of participants' personality traits.

${ }^{6} \mathrm{HE}+\mathrm{HN} M=3.98(S D=.88) ; \mathrm{HE}+\mathrm{LN} M=4.13(S D=.88) ; \mathrm{LE}+\mathrm{HN} M=3.49(S D=.80) ; \mathrm{LE}+\mathrm{LN} M=3.56(S D=.76)$. ${ }^{7} \mathrm{HE}+\mathrm{HN} M=4.17(S D=1.11) ; \mathrm{LE}+\mathrm{HN} M=4.15(S D=1.13) ; \mathrm{HE}+\mathrm{LN} M=3.16(S D=.89) ; \mathrm{LE}+\mathrm{LN} M=3.08(S D=$ .89).
} 


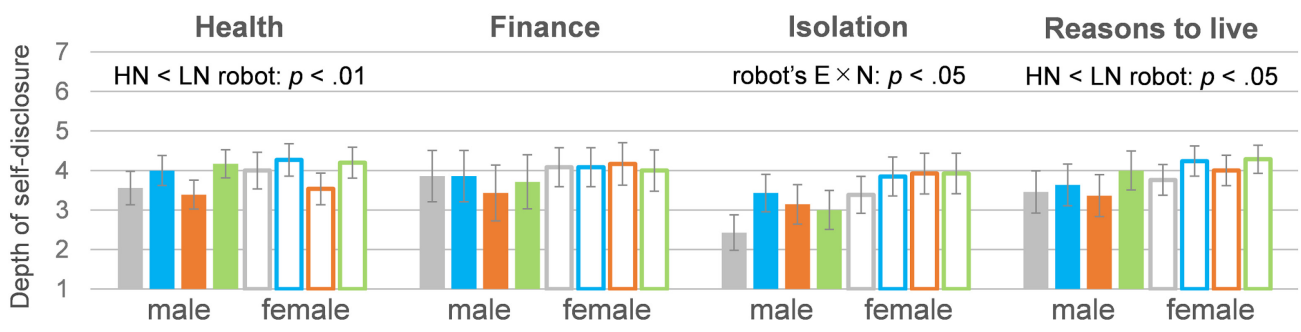

(a) $\mathrm{HE}+\mathrm{HN}$ participants

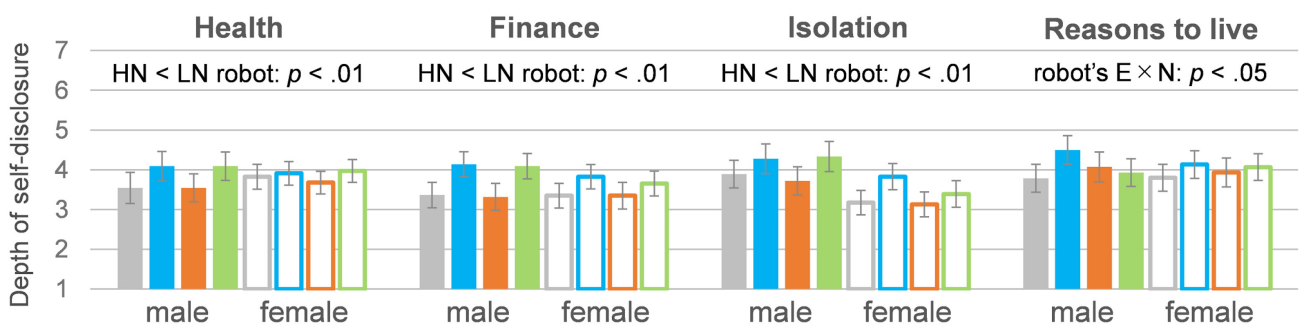

(b) HE+LN participants

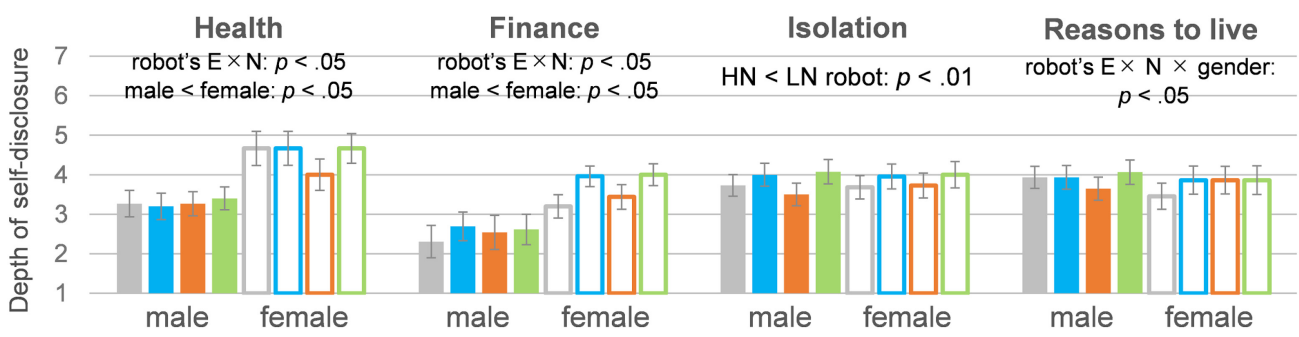

(c) LE+HN participants

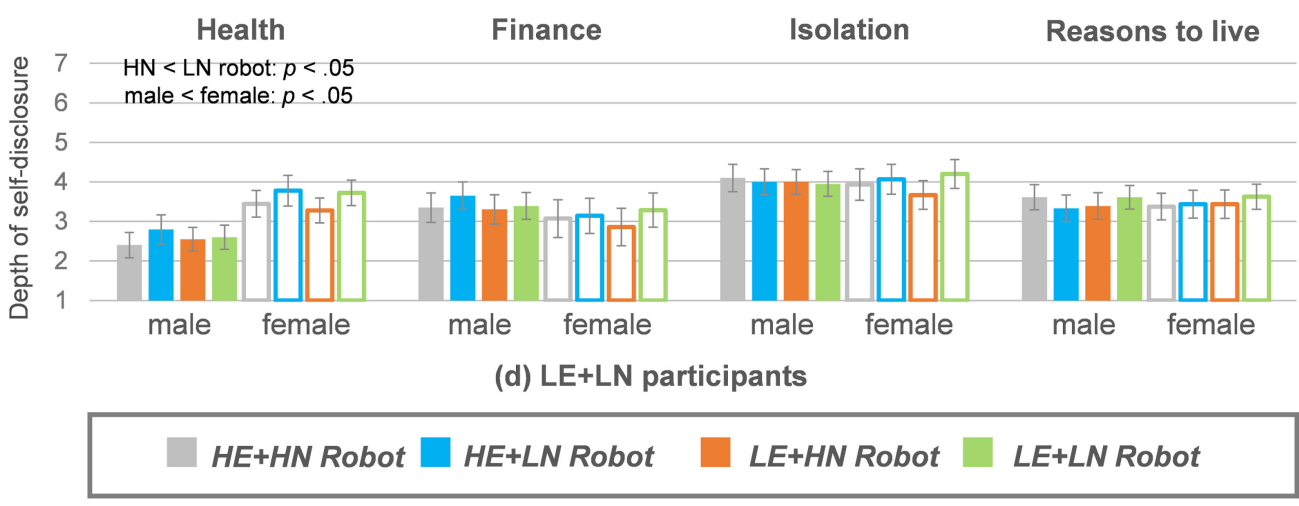

Fig. 6. The depth of self-disclosure measured from participants against four types of robots with different personality traits. "HE+HN Robot" denotes the robot having high extroversion trait (HE) and high neuroticism trait (HN), whereas "LE+LN participants" denotes the participants having low extroversion trait (LE) and low neuroticism trait (LN). The results are divided by four kinds of topics chosen initially by participants at the time of their recruitment: health, finance, isolation, and reasons to live. 

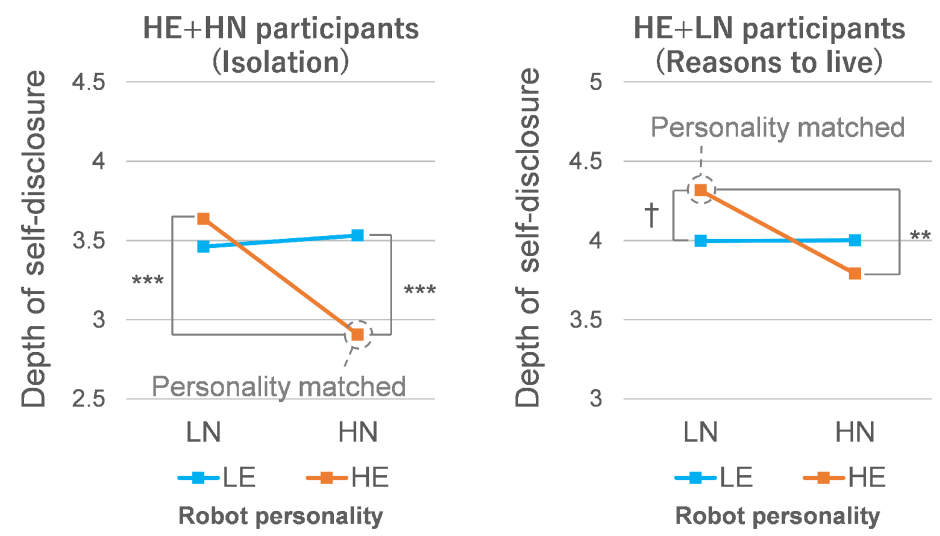

Fig. 7. Matching robot personality to participants with high extroversion. Similarity-attraction was observed in the participants with low neuroticism ( $\mathrm{HE}+\mathrm{LN}$ participants), whereas similarity-repulsion was observed in the participants with high neuroticism ( $\mathrm{HE}+\mathrm{HN}$ participants).

was larger than the case with HN robots in topics concerning health $(F(1,31)=7.65, p=.009$, $\left.\eta_{p}{ }^{2}=.20\right)$ and reasons to live $\left(F(1,30)=5.44, p=.027, \eta_{p}{ }^{2}=.15\right)$.

There was also a significant interaction between two personality traits (extroversion and neuroticism) of the robots in topics concerning isolation $\left(F(1,25)=4.88, p=.037, \eta_{p}^{2}=.16\right)$. Then, post hoc comparisons using $t$-tests showed significant differences in the average depth of selfdisclosure between the robot with high extroversion and high neuroticism $(\mathrm{HE}+\mathrm{HN}$ robot: matched to the $\mathrm{HE}+\mathrm{HN}$ participants, $\left.M^{*}=2.91, S D^{*}=1.68^{8}\right)$ and the $\mathrm{LE}+\mathrm{HN} \operatorname{robot}\left(M^{*}=3.53, S D^{*}=1.86\right.$; $t(60)=3.88, p<.001, d=.35)$, as well as HE+LN robot $\left(M^{*}=3.64, S D^{*}=1.78 ; t(60)=3.65\right.$, $p<.001, d=.42$ ), which is illustrated in Figure 7 (left).

5.7.3 HE+LN Participants. The marginal means of the depth of self-disclosure obtained from the $\mathrm{HE}+\mathrm{LN}$ participants are shown in Figure 6(b). There was a significant main effect of the neuroticism trait of the robots in three kinds of topics: the depth of self-disclosure to LN robots was larger than the case with $\mathrm{HN}$ robots in topics concerning health $\left(F(1,54)=8.80, p=.004, \eta_{p}^{2}=.14\right)$, finance $\left(F(1,43)=19.6, p<.001, \eta_{p}{ }^{2}=.31\right)$, and isolation $\left(F(1,39)=8.11, p=.007, \eta_{p}{ }^{2}=.17\right)$.

There was also a significant interaction between two personality traits (extroversion and neuroticism) of the robots in topics concerning reasons to live $\left(F(1,27)=5.73, p=.024, \eta_{p}{ }^{2}=.18\right.$ ). Then, post hoc comparisons showed a significant difference in the average depth of self-disclosure between the robot with high extroversion and low neuroticism (HE+LN robot: matched to the $\mathrm{HE}+\mathrm{LN}$ participants, $\left.M^{*}=4.32, S D^{*}=1.35\right)$ and the $\mathrm{HE}+\mathrm{HN} \operatorname{robot}\left(M^{*}=3.79, S D^{*}=1.32 ; t(54)=\right.$ $3.14, p=.003, d=.39$ ), and a marginally significant difference between the $\mathrm{HE}+\mathrm{LN}$ robot and the $\mathrm{LE}+\mathrm{LN}$ robot $\left(M^{*}=4.00, S D^{*}=1.30 ; t(54)=1.97, p=.054, d=.24\right)$, which is illustrated in Figure 7 (right).

5.7.4 LE+HN Participants. The marginal means of the depth of self-disclosure obtained from the $\mathrm{LE}+\mathrm{HN}$ participants are shown in Figure 6(c). There was a significant main effect of the neuroticism trait of the robots: the depth of self-disclosure to LN robots was larger than the case with $\mathrm{HN}$ robots in topics concerning isolation $\left(F(1,46)=11.0, p=.002, \eta_{p}{ }^{2}=.19\right)$. There was a significant main effect of the gender of the participants in two kinds of topics: the depth of self-disclosure in female

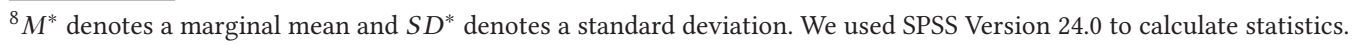




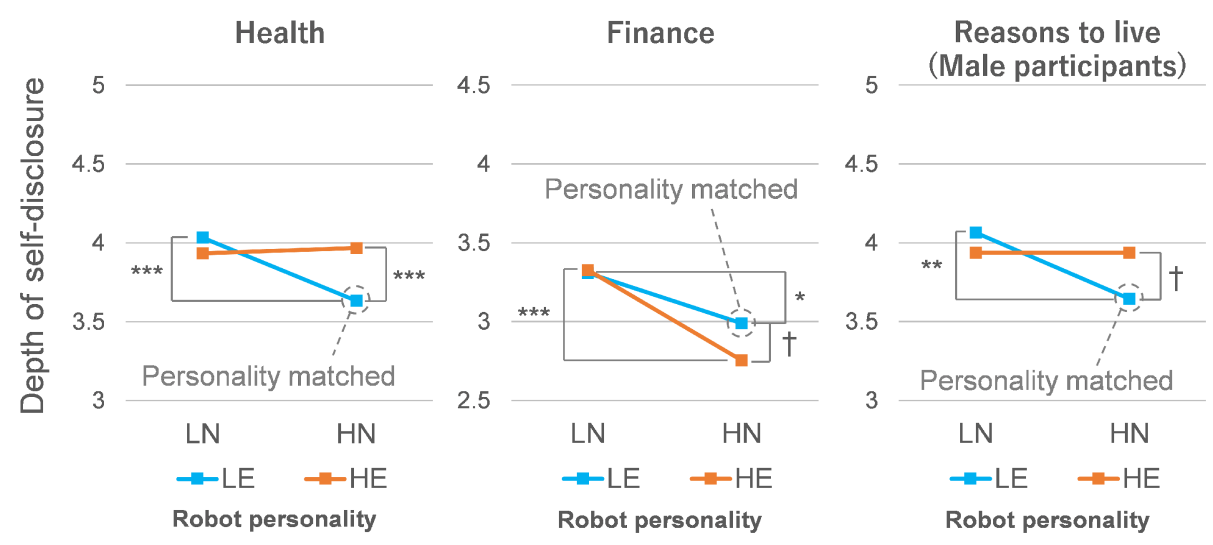

Fig. 8. Matching robot personality to participants with low extroversion and high neuroticism.

participants was larger than that in male participants in topics concerning health $(F(1,22)=6.82$, $\left.p=.016, \eta_{p}^{2}=.24\right)$ and finance $\left(F(1,36)=6.02, p=.019, \eta_{p}^{2}=.14\right)$.

There was a significant interaction between two personality traits (extroversion and neuroticism) of the robots in two kinds of topics concerning health $\left(F(1,22)=5.67, p=.026, \eta_{p}^{2}=.21\right)$ and finance $\left(F(1,36)=4.23, p=.047, \eta_{p}^{2}=.11\right)$. Then, we performed post hoc comparisons. In the health topics, significant differences were found in the average depth of self-disclosure between the robot with low extroversion and high neuroticism ( $\mathrm{LE}+\mathrm{HN}$ robot: matched to the $\mathrm{LE}+\mathrm{HN}$ participants, $\left.M^{*}=3.63, S D^{*}=1.22\right)$ and the $\mathrm{HE}+\mathrm{HN} \operatorname{robot}\left(M^{*}=3.97, S D^{*}=1.34 ; t(44)=2.04, p=.047\right.$, $d=.26)$, as well as the LE+LN $\operatorname{robot}\left(M^{*}=4.03, S D^{*}=1.16 ; t(44)=2.27, p=.028, d=.34\right)$, which is illustrated in Figure 8 (Health). Next, in the finance topics, two significant differences and a marginally significant difference in the average depth of self-disclosure were found as illustrated in Figure 8 (Finance): a significant difference between the robot with low extroversion and high neuroticism $\left(\mathrm{LE}+\mathrm{HN}\right.$ robot: matched to the $\mathrm{LE}+\mathrm{HN}$ participants, $\left.M^{*}=2.99, S D^{*}=1.64\right)$ and the $\mathrm{LE}+\mathrm{LN}$ robot $\left(M^{*}=3.31, S D^{*}=1.46 ; t(72)=2.22, p=.030, d=.21\right)$, a significant difference between the $\mathrm{HE}+\mathrm{HN}$ robot $\left(M^{*}=2.75, S D^{*}=1.56\right)$ and the $\mathrm{HE}+\mathrm{LN}$ robot $\left(M^{*}=3.33, S D^{*}=1.38\right.$; $t(72)=3.99, p<.001, d=.39)$, and a marginally significant difference between the LE+HN robot and the $\mathrm{HE}+\mathrm{HN}$ robot $(t(72)=1.71, p=.092, d=.05)$.

There was also a significant three-way interaction between two personality traits (extroversion and neuroticism) of the robots and the gender of the participants in topics concerning reasons to live $\left(F(1,51)=6.18, p=.016, \eta_{p}{ }^{2}=.11\right)$. Then, we performed a simple interaction test on this topic. For male participants, a marginally significant interaction was found between two personality traits of robot (extroversion and neuroticism) $\left(F(1,51)=3.82, p=.056, \eta_{p}{ }^{2}=.14\right)$, whereas no significant interaction was found in female participants $\left(F(1,51)=2.58, p=.11, \eta_{p}{ }^{2}=.086\right)$. For more detail, $t$-tests showed that male participants self-disclosed on a deeper level to the HE+HN robot $\left(M^{*}=\right.$ $3.94, S D^{*}=1.56$ ) than to the $\mathrm{LE}+\mathrm{HN}$ robot (matched to the $\mathrm{LE}+\mathrm{HN}$ participants, $M^{*}=3.65, S D^{*}=$ $1.63 ; t(51)=1.91, p=.061, d=.18)$. At the same time, in this condition, male participants selfdisclosed on a deeper level to the $\mathrm{LE}+\mathrm{LN}$ robot $\left(M^{*}=4.07, S D^{*}=1.71\right)$ than to the LE+HN robot $(t(51)=2.76, p=.008, d=.25)$ (on the right side in Figure 8).

5.7.5 LE+LN Participants. The marginal means of the depth of self-disclosure obtained from the LE+LN participants are shown in Figure 6(d). There was a significant main effect of the neuroticism trait of the robots: the depth of self-disclosure to LN robots was larger than the case with $\mathrm{HN}$ robots in topics concerning health $\left(F(1,36)=6.27, p=.017, \eta_{p}{ }^{2}=.15\right)$. There was also a significant main 
effect of the gender of the participants: the depth of self-disclosure in female participants was larger than that in male participants in the health topics $\left(F(1,36)=5.36, p=.026, \eta_{p}{ }^{2}=.13\right)$.

5.7.6 Self-Esteem of Participants. Correlation coefficients were calculated between the scores of self-esteem and personality traits of participants. Significant correlations were confirmed $(p<$ .001; extroversion: $r=.31$, and neuroticism: $r=.47)$. A one-way ANOVA showed a significant difference in the scores of self-esteem between the personality traits $(F(3,585)=35.7, p<.001$, $\left.\eta_{p}{ }^{2}=.16\right)$. Then, from a Tukey HSD analysis, we found that the scores of self-esteem divided by the four participant groups were ranked as $L E+H N<H E+H N<L E+L N<H E+L N(p<.05)$.

\subsection{Discussions}

The results obtained from the HE+HN participants (Section 5.7.2) show that the participants in this group self-disclosed deeper to LN robots than to HN robots in topics concerning health and reasons to live. In addition, if their personality traits $(\mathrm{HE}+\mathrm{HN})$ match the ones of the robot $(\mathrm{HE}+\mathrm{HN}$ robot), the self-disclosure of the participants was suppressed in isolation topics. Therefore, we can interpret that, in this group (except the case with finance topics), the depth of self-disclosure decreased due to a similarity-repulsion effect, which supports hypothesis H2b.

Eysenck's portrayal of a person with high neuroticism was "anxious and afraid when confronted with social situations, seeks to avoid them in order to escape from this negative feeling, but frequently wishes that he could be more sociable" [15]. This negative feeling may weaken the person's self-esteem. In fact, the results reported in Section 5.7.6 can be interpreted in that participants with higher neuroticism had lower self-esteem. The similarity-repulsion could have arisen from this negative feeling in watching the robot.

The results obtained from the HE+LN participants (Section 5.7.3) show that the participants in this group self-disclosed on a deeper level to LN robots than to HN robots in topics concerning health, finance, and isolation. In addition, if their personality traits $(\mathrm{HE}+\mathrm{LN})$ match the ones of the robot (HE+LN robot), the self-disclosure of the participants was increased in reasons to live topics. Therefore, we can interpret that, in this group, the depth of self-disclosure increased due to a similarity-attraction effect, which supports hypothesis H2a.

The results obtained from the LE+HN participants (Section 5.7.4) show gender differences: in two kinds of topics concerning health and finance, female participants self-disclosed on a deeper level than male participants. In addition, the participants in this group self-disclosed on a deeper level to $\mathrm{LN}$ robots than to $\mathrm{HN}$ robots in topics concerning isolation. In the other topics, the results showed complicated interactions as summarized in Figure 8: similarity-repulsion was observed in health topics, whereas results were mixed in finance topics. Furthermore, in reasons to live topics, again, there was a gender difference, a three-way interaction between personality traits of the robots and the gender of the participants. Therefore, we can interpret that in this group (except the case with finance topics and some cases with female participants), the depth of self-disclosure decreased due to a similarity-repulsion effect, which supports hypothesis H2b.

Again, according to the Eysenck's portrayal of a person with high neuroticism, the person was "anxious and afraid when confronted with social situations, seeks to avoid them in order to escape from this negative feeling, but frequently wishes that he could be more sociable” [15]. In addition, it was discussed that there was a difference between extroverts and introverts in the degree of craving excitement in social situations [16]. Therefore, introverts may not expect their subjective rewards such as excitement or pleasure obtained through interacting with robots compared to extroverts. The LE $+\mathrm{HN}$ participants were introverted, and they could have flexibly changed their behaviors (self-disclosure) according to the type of robots and topics so that they would not be hurt. 
Table 3. Results Summary of Study 2, Together with Recommendations for Designing the Personality Traits of Mediator Robots

\begin{tabular}{|c|c|c|c|c|c|}
\hline \multirow{2}{*}{\multicolumn{2}{|c|}{ Topic }} & \multicolumn{4}{|c|}{ User's Personality Traits } \\
\hline & & $\mathrm{HE}+\mathrm{HN}$ & $\mathrm{HE}+\mathrm{LN}$ & $\mathrm{LE}+\mathrm{HN}$ & $\mathrm{LE}+\mathrm{LN}$ \\
\hline \multirow[t]{2}{*}{ Health } & Matching Effect & - & - & Repulsive & - \\
\hline & Recommended Design & $\mathrm{LN}$ & $\mathrm{LN}$ & Unmatched & $\mathrm{LN}$ \\
\hline \multirow[t]{2}{*}{ Finance } & Matching Effect & - & - & Mixed & - \\
\hline & Recommended Design & - & $\overline{\mathrm{LN}}$ & $\overline{\mathrm{LN}}-\overline{-}$ & - \\
\hline \multirow[t]{2}{*}{ Isolation } & Matching Effect & Repulsive & - & - & - \\
\hline & Recommended Design & Unmatched & $\overline{\mathrm{L}}$ & $\overline{\mathrm{LN}}$ & - \\
\hline \multirow[t]{2}{*}{ Reasons to live } & Matching Effect & - & Attractive & Repulsive (male) & - \\
\hline & Recommended Design & $\overline{\mathrm{LN}}$ & Matched & Unmatched (male) & - \\
\hline
\end{tabular}

Finally, in the LE+LN participants group (Section 5.7.5), significant differences were found only in the health topic: the participants self-disclosed on a deeper level to LN robots than to HN robots, and female participants self-disclosed on a deeper level than male participants. Overall, in this participants group, the depth of self-disclosure was not affected much by robot personality, which could have been due to the emotional stability (low extroversion and low neuroticism) of the participants. The participants in this group were also introverted, and they might have felt little excitement in interacting with the robots. At the same time, because their neuroticism was also low, they might have had little anxiety as well.

To conclude, Table 3 summarizes the results of study 2 and recommendations for designing the personality traits of mediator robots, which answers the second research question in this paper, "RQ2: How should the robot's character be personalized to the elderly user?" In previous HRI studies, matching robot personality to the user had been discussed mainly in a single dimension (especially extroversion) $[2,5,33,38,55]$. In contrast, the present study 2 made a further detailed investigation involving extroversion, neuroticism, and human gender.

\section{GENERAL DISCUSSIONS}

\subsection{Summary of Two Studies}

In study 1 , we explored dialogue topics in which social mediator robots could well encourage the self-disclosure of elderly people. The results suggest that in topics concerning loss experience, introducing social mediator robots can be particularly effective compared to direct conversations over phones. By using the social mediator robots, the elderly person's hesitation or psychological resistance in self-disclosure could be alleviated. A possible reason would be due to the avoidance of receiving negative feedback directly from recipients. In addition, as Uchida et al. [58] suggested, since robots do not share social statements with humans, disclosers can self-disclose with little concern of receiving negative feedback from the mediator robots. Therefore, although it is necessary for mediator robots to be regarded as social others, elderly self-disclosure may be suppressed if the robots are too much like humans.

In study 2, we discussed personality factors between mediator robots and the users, and then we reported detailed findings and recommendations for designing the personality traits of the mediator robots (Table 3). However, we often reported main effects concerning the neuroticism trait of the mediator robots. Overall, the results show that participants self-disclose on a deeper level to LN robots than to $\mathrm{HN}$ robots. Low neuroticism may be a trait that is generally effective for mediator robots. In contrast, we could not find as many main effects regarding the extroversion trait of mediator robots compared to the neuroticism trait. 


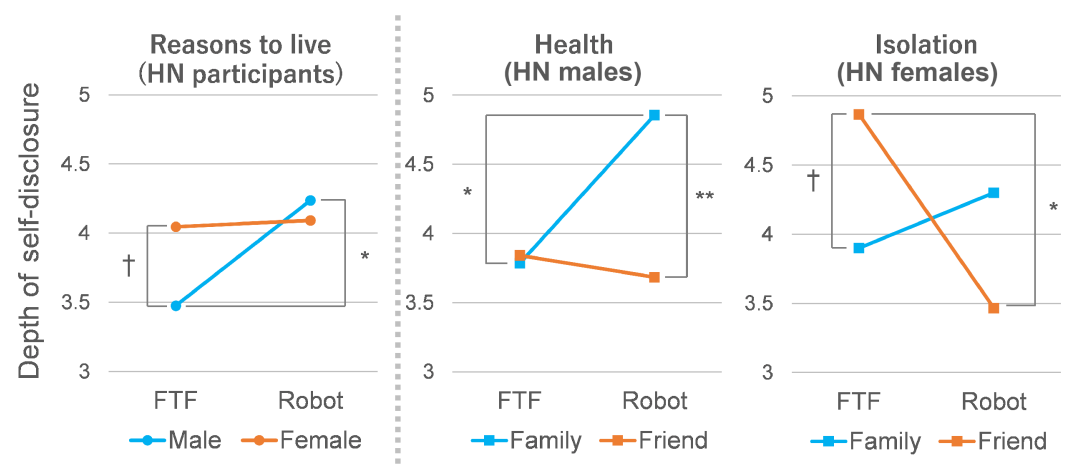

Fig. 9. Gender differences in disclosers regarding the depth of self-disclosure. FTF, face-to-face condition; Robot, robot condition.

The results of study 2 also show that the personality traits of participants may greatly affect the HRI discussed in this study. For example, we observed very little effect in the LE+LN participants group. The study suggests that when measuring human self-disclosure, we should take into consideration multiple personality traits of participants.

\subsection{Gender Factors of Disclosers}

We conducted a follow-up study about gender differences in participants. Following the literature in CMC [3], we compared the participants' depth of self-disclosure between a robot condition and a face-to-face condition. In the robot condition, participants were instructed to use a mediator robot in self-disclosure, whereas in the face-to-face condition, participants were instructed to talk to a human recipient face-to-face.

We collected data from the participants of study $2(N=589)$ and measured their depth of selfdisclosure across the two conditions. In the robot condition, participants watched a short video in which a recipient person receives a message from the Type-A robot (Section 3.2).

A $2 \times 2 \times 2$ split-plot factorial ANOVA with a within-participant factor (robot/face-to-face) and two between-participant factors, participant's gender (male/female) and recipient types (family/friend) was conducted on four kinds of topics (health/finance/isolation/reasons to live) and two personality traits (high/low neuroticism) of the participants.

In the $\mathrm{HN}$ participants group, there was a significant interaction between conditions and gender in topics concerning reasons to live $\left(F(1,81)=3.97, p=.050, \eta_{p}{ }^{2}=.047\right)$. Post hoc comparisons using $t$-tests showed a significant difference in the average depth of self-disclosure of the male HN participants between the robot condition $\left(M^{*}=4.22, S D^{*}=1.45\right)$ and the face-to-face condition $\left(M^{*}=3.47, S D^{*}=1.45 ; t(81)=3.00, p=.004, d=.52\right)$, and a marginally significant difference between female $\mathrm{HN}$ participants $\left(M^{*}=4.06, S D^{*}=1.44\right)$ and male $\mathrm{HN}$ participants in the face-to-face condition $(t(162)=1.87, p=.063, d=.41)$ (Figure 9, left).

There were also significant three-way interactions between conditions, gender, and recipient types in topics concerning health $\left(F(1,53)=5.34, p=.025, \eta_{p}^{2}=.091\right)$ and isolation $(F(1,71)=$ $\left.4.62, p=.035, \eta_{p}^{2}=.061\right)$ in the HN participants group. Simple interaction tests showed a significant interaction between conditions and recipient types in male $\mathrm{HN}$ participants in topics concerning health $\left(F(1,31)=4.10, p=.048, \eta_{p}^{2}=.095\right)$, and another significant interaction between conditions and recipient types in female $\mathrm{HN}$ participants in topics concerning isolation $\left(F(1,33)=6.25, p=.015, \eta_{p}{ }^{2}=.18\right)$. Then, in topics concerning health, it was found that male HN participants self-disclose on a deeper level to family recipients in the robot condition $\left(M^{*}=4.86\right.$, 
$\left.S D^{*}=1.56\right)$ than in the face-to-face condition $\left(M^{*}=3.79, S D^{*}=1.61 ; t(53)=2.33, p=.024\right.$, $d=.68)$, and they also self-disclose on a deeper level to family recipients than to friend recipients $\left(M^{*}=3.68, S D^{*}=1.56 ; t(53)=2.73, p=.009, d=.75\right)$ in the robot condition (Figure 9, center). In contrast, in topics concerning isolation, female $\mathrm{HN}$ participants self-disclose on a deeper level to friend recipients in the face-to-face condition $\left(M^{*}=4.87, S D^{*}=1.65\right)$ than in the robot condition $\left(M^{*}=3.47, S D^{*}=1.67 ; t(71)=2.57, p=.012, d=.84\right)$, and they also tend to self-disclose on a deeper level to friend recipients than to family recipients $\left(M^{*}=3.90, S D^{*}=1.65\right)$ in the face-toface condition $(t(71)=1.90, p=.062, d=.59)$ (Figure 9, right).

The results show some opposite tendencies between male and female participants. We may interpret the results by suggesting that the mediator robot is the most effective for male participants with high neuroticism in dialogue topics concerning reasons to live and health (to family recipients). In addition, the mediator robot may not be that effective for female participants with high neuroticism in a dialogue topic concerning isolation. However, for the LN participants group, no significant effect was found in any factor.

\subsection{Overall Limitations and Future Works}

Across two studies reported in this article, a fundamental limitation exists in that both studies lack a behavioral measurement of self-disclosure. We used questionnaires and did not directly measure the disclosing behaviors (i.e., utterances) of participants. In addition, all measurements were conducted within relatively short time windows. Another big issue was that our studies depended on simulated conversation scenarios. In addition, the scripts we used were just an example, and we cannot claim generality. In study 2, video stimuli were also used. Empirical HRI studies are awaited to strengthen the results of those studies. Finally, all participants in these studies were Japanese. There must have been some cultural factors that might have affected the results.

The studies reported in this article mainly discussed mediator robots and disclosers. Further studies are needed to take the recipient side into consideration. In fact, one participant stated, "I think I cannot tell such a trouble to my friend. I'm worried if the robot could separate my friend from me." Another stated, "I feel telling my trouble through this robot might be rude for my daughter." These comments imply a need for designing social mediator robots by considering not only the discloser side but also the recipient side.

\section{CONCLUSION}

For designing effective social mediator robots that encourage elderly people to self-disclose to prevent them from being socially isolated, we presented the results from two studies investigating dialogue topics and the personality traits of mediator robots and disclosers. Design recommendations were discussed and summarized based on the results.

\section{ACKNOWLEDGMENTS}

We appreciate editors and reviewers for their detailed reviews and very useful comments.

\section{REFERENCES}

[1] Irwin Altman and Dalmas A. Taylor. 1973. Social Penetration: The Development of Interpersonal Relationships. Holt, Rinehart \& Winston.

[2] Amir Aly and Adriana Tapus. 2013. A model for synthesizing a combined verbal and nonverbal behavior based on personality traits in human-robot interaction. In Proceedings of the 8th ACM/IEEE International Conference on HumanRobot Interaction. IEEE, Los Alamitos, CA, 325-332. DOI : https://doi.org/10.1109/HRI.2013.6483606

[3] Yair Amichai-Hamburger, Galit Wainapel, and Shaul Fox. 2002. "On the Internet no one knows I'm an introvert": Extroversion, neuroticism, and Internet interaction. CyberPsychology \& Behavior 5, 2 (2002), 125-128. DOI: https: //brkdoi.org/10.1089/109493102753770507 
[4] Fatemeh Amirazodi and Maryam Amirazodi. 2011. Personality traits and self-esteem. Procedia: Social and Behavioral Sciences 29 (2011), 713-716. DOI : https://doi.org/10.1016/j.sbspro.2011.11.296

[5] Sean Andrist, Bilge Mutlu, and Adriana Tapus. 2015. Look like me: Matching robot personality via gaze to increase motivation. In Proceedings of the 33rd Annual ACM Conference on Human Factors in Computing Systems. ACM, New York, NY, 3603-3612. DOI : https://doi.org/10.1145/2702123.2702592

[6] Sean Andrist, Xiang Zhi Tan, Michael Gleicher, and Bilge Mutlu. 2014. Conversational gaze aversion for humanlike robots. In Proceedings of the 2014 ACM/IEEE International Conference on Human-Robot Interaction. ACM, New York, NY, 25-32. DOI : https://doi.org/10.1145/2559636.2559666

[7] Roy F. Baumeister and Kathleen D. Vohs (Eds.). 2007. Encyclopedia of Social Psychology. Sage. D0I : https://doi.org/10. $4135 / 9781412956253$

[8] Elizabeth Broadbent, Chandimal Jayawardena, Ngaire Kerse, Rebecca Q. Stafford, and Bruce A. MacDonald. 2011. Human-robot interaction research to improve quality of life in elder care-An approach and issues. In Proceedings of the Workshops at the 25th AAAI Conference on Artificial Intelligence. https://www.aaai.org/ocs/index.php/WS/ AAAIW11/paper/view/3875/4279.

[9] Franziska Burger, Joost Broekens, and Mark A. Neerincx. 2017. Fostering relatedness between children and virtual agents through reciprocal self-disclosure. In BNAIC 2016: Artificial Intelligence. Communications in Computer and Information Science, Vol. 765. Springer, Cham, Switzerland, 137-154. DOI : https://doi.org/10.1007/978-3-319-67468-1_ 10

[10] Colleen M. Carpinella, Alisa B. Wyman, Michael A. Perez, and Steven J. Stroessner. 2017. The Robotic Social Attributes Scale (RoSAS): Development and validation. In Proceedings of the 2017 ACM/IEEE International Conference on HumanRobot Interaction. ACM, New York, NY, 254-262. DOI : https://doi.org/10.1145/2909824.3020208

[11] Nikolas Coupland, Justine Coupland, Howard Giles, and Karen Henwood. 1988. Accommodating the elderly: Invoking and extending a theory. Language in Society 17, 1 (1988), 1-41. DOI : https://doi.org/10.1017/S0047404500012574

[12] Ronen Cuperman and William Ickes. 2009. Big five predictors of behavior and perceptions in initial dyadic interactions: Personality similarity helps extraverts and introverts, but hurts "disagreeables." fournal of Personality and Social Psychology 97, 4 (2009), 667-684. DOI : https://doi.org/10.1037/a0015741

[13] Erik H. Erikson and Joan M. Erikson. 1998. The Life Cycle Completed (Extended Version). W. W. Norton \& Company.

[14] Erik H. Erikson, Joan M. Erikson, and Helen Q. Kivnick. 1986. Vital Involvement in Old Age: The Experience of Old Age in Our Time. W. W. Norton \& Company.

[15] Hans J. Eysenck. 1956. The questionnaire measurement of neuroticism and extraversion. Revista di Psicologia 50 (1956), 113-140.

[16] Hans J. Eysenck and Sybil B. G. Eysenck. 1975. Manual of the Eysenck Personality Questionnaire (Funior and Adult). Hodder \& Stoughton.

[17] Julia Fink. 2012. Anthropomorphism and human likeness in the design of robots and human-robot interaction. In Social Robotics. Lecture Notes in Computer Science, Vol. 7621. Springer, 199-208. DOI:https://doi.org/10.1007/ 978-3-642-34103-8_20

[18] David C. Funder and Carl D. Sneed. 1993. Behavioral manifestations of personality: An ecological approach to judgmental accuracy. Journal of Personality and Social Psychology 64, 3 (1993), 479-490. DOI : https://doi.org/10.1037/ 0022-3514.64.3.479

[19] Adrian Furnham. 1990. Language and personality. In Handbook of Language and Social Psychology, H. Giles and W. P. Robinson (Eds.). John Wiley \& Sons, 73-95.

[20] Jonathan Gratch, Ning Wang, Jillian Gerten, Edward Fast, and Robin Duffy. 2007. Creating rapport with virtual agents. In Intelligent Virtual Agents. Lecture Notes in Computer Science, Vol. 4722. Springer, 125-138. DOI : https://doi.org/ 10.1007/978-3-540-74997-4_12

[21] Barbara K. Haight. 1988. The therapeutic role of a structured life review process in homebound elderly subjects. fournal of Gerontology 43, 2 (1988), 40-44. DOI : https://doi.org/10.1093/geronj/43.2.P40

[22] Marcel Heerink, Ben Kröse, Vanessa Evers, and Bob Wielinga. 2010. Assessing acceptance of assistive social agent technology by older adults: The Almere model. International fournal of Social Robotics 2 (2010), 361-375. DOI : https:// doi.org/10.1007/s12369-010-0068-5

[23] Atsushi Hiyama, Akihiro Kosugi, Kentarou Fukuda, Masatomo Kobayashi, and Michitaka Hirose. 2017. Facilitating remote communication between senior communities with telepresence robots. In Human Aspects of IT for the Aged Population: Applications, Services and Contexts. Lecture Notes in Computer Science, Vol. 10298. Springer, Cham, Switzerland, 501-515. DOI : https://doi.org/10.1007/978-3-319-58536-9_40

[24] James S. House. 1981. Work Stress and Social Support. Addison-Wesley.

[25] Oliver P. John and Sanjay Srivastava. 1999. The big five trait taxonomy: History, measurement, and theoretical perspectives. In Handbook of Personality, Second Edition: Theory and Research, L. A. Pervin and O. P. John (Eds.). Guilford, 102-138. 
[26] Adam N. Joinson. 2001. Self-disclosure in computer-mediated communication: The role of self-awareness and visual anonymity. European fournal of Social Psychology 31, 2 (2001), 177-192. DOI : https://doi.org/10.1002/ejsp.36

[27] Sidney M. Jourard. 1971. The Transparent Self. Van Nostrand Reinhold.

[28] Michael H. Kernis. 2003. Toward a conceptualization of optimal self-esteem. Psychological Inquiry 14, 1 (2003), 1-26. DOI : https://doi.org/10.1207/S15327965PLI1401_01

[29] Toru Kobayashi, Kazushige Katsuragi, Taishi Miyazaki, and Kenichi Arai. 2017. Social media intermediation robot for elderly people using external cloud-based services. In Proceedings of the 5th IEEE International Conference on Mobile Cloud Computing, Services, and Engineering. IEEE, Los Alamitos, CA, 31-38. DOI : https://doi.org/10.1109/ MobileCloud.2017.18

[30] Saso Koceski and Natasa Koceska. 2016. Evaluation of an assistive telepresence robot for elderly healthcare. Fournal of Medical Systems 40 (2016), Article No. 121. DOI : https://doi.org/10.1007/s10916-016-0481-x

[31] Hirokazu Kumazaki, Zachary Warren, Amy Swanson, Yuichiro Yoshikawa, Yoshio Matsumoto, Hideyuki Takahashi, Nilanjan Sarkar, et al. 2018. Can robotic systems promote self-disclosure in adolescents with autism spectrum disorder? A pilot study. Frontiers in Psychiatry 9 (2018), Article No. 36. DOI : https://doi.org/10.3389/fpsyt.2018.00036

[32] Jun Ki Lee and Cynthia Breazeal. 2010. Human social response toward humanoid robot's head and facial features. In CHI '10 Extended Abstracts on Human Factors in Computing Systems. ACM, New York, NY, 4237-4242. DOI : https:// doi.org/10.1145/1753846.1754132

[33] Kwan Min Lee and Clifford Nass. 2003. Designing social presence of social actors in human computer interaction. In Proceedings of the SIGCHI Conference on Human Factors in Computing Systems. ACM, New York, NY, $289-296$. DOI : https://doi.org/10.1145/642611.642662

[34] Richard Lippa. 1998. The nonverbal display and judgment of extraversion, masculinity, femininity, and gender diagnosticity: A lens model analysis. Journal of Research in Personality 32, 1 (1998), 80-107. DOI : https://doi.org/10.1006/ jrpe.1997.2189

[35] Michal Luria, Jessica Hodgins, and Jodi Forlizzi. 2018. The effects of eye design on the perception of social robots. In Proceedings of the 27th IEEE International Conference on Robot and Human Interactive Communication. IEEE, Los Alamitos, CA, 1032-1037. DOI : https://doi.org/10.1109/ROMAN.2018.8525767

[36] Stephen M. Marson and Rasby M. Powell. 2014. Goffman and the infantilization of elderly persons: A theory in development. Journal of Sociology and Social Welfare 41, 4 (2014), 143-158.

[37] Nikolas Martelaro, Victoria C. Nneji, Wendy Ju, and Pamela Hinds. 2016. Tell me more: Designing HRI to encourage more trust, disclosure, and companionship. In Proceedings of the 11th ACM/IEEE International Conference on HumanRobot Interaction. IEEE, Los Alamitos, CA, 181-188. DOI : https://doi.org/10.1109/HRI.2016.7451750

[38] Alexandros Mileounis, Raymond H. Cuijpers, and Emilia I. Barakova. 2015. Creating robots with personality: The effect of personality on social intelligence. In Artificial Computation in Biology and Medicine. Lecture Notes in Computer Science, Vol. 9107. Springer, 119-132. DOI : https://doi.org/10.1007/978-3-319-18914-7_13

[39] Youngme Moon. 2000. Intimate exchanges: Using computers to elicit self-disclosure from consumers. fournal of Consumer Research 26, 4 (2000), 323-339. DOI : https://doi.org/10.1086/209566

[40] Tsutomu Namikawa, Iori Tani, Takafumi Wakita, Ryuichi Kumagai, Ai Nakane, and Hiroyuki Noguchi. 2012. Development of a short form of the Japanese big-five scale, and a test of its reliability and validity. Japanese fournal of Psychology 83, 2 (2012), 91-99 (in Japanese). DOI : https://doi.org/10.4992/jjpsy.83.91

[41] Clifford Nass and Kwan Min Lee. 2001. Does computer-synthesized speech manifest personality? Experimental tests of recognition, similarity-attraction, and consistency-attraction. Fournal of Experimental Psychology: Applied 7, 3 (2001), 171-181. DOI : https://doi.org/10.1037/1076-898X.7.3.171

[42] Clifford Nass, Youngme Moon, B. J. Fogg, Byron Reeves, and D. Christopher Dryer. 1995. Can computer personalities be human personalities? International fournal of Human-Computer Studies 43, 2 (1995), 223-239. DOI : https://doi.org/ 10.1006/ijhc.1995.1042

[43] Yohei Noguchi and Fumihide Tanaka. 2017. A pilot study investigating self-disclosure by elderly participants in agentmediated communication. In Proceedings of the 26th IEEE International Symposium on Robot and Human Interactive Communication. IEEE, Los Alamitos, CA, 29-34. DOI : https://doi.org/10.1109/ROMAN.2017.8172276

[44] Erina Okamura and Fumihide Tanaka. 2016. A pilot study about remote teaching by elderly people to children over a two-way telepresence robot system. In Proceedings of the 11th ACM/IEEE International Conference on Human-Robot Interaction. IEEE, Los Alamitos, CA, 489-490. DOI : https://doi.org/10.1109/HRI.2016.7451820

[45] Atsushi Oshio, Shingo Abe, and Pino Cutrone. 2012. Development, reliability, and validity of the Japanese version of ten item personality inventory (TIPI-J). fapanese fournal of Personality 21, 1 (2012), 40-52 (in Japanese). DOI : https:// doi.org/10.2132/personality.21.40

[46] Laurel D. Riek. 2016. Robotics technology in mental health care. In Artificial Intelligence in Behavioral and Mental Health Care, D. D. Luxton (Ed.). Academic Press, 185-203. DOI : https://doi.org/10.1016/B978-0-12-420248-1.00008-8 
[47] Ben Robins, Kerstin Dautenhahn, and Paul Dickerson. 2009. From isolation to communication: A case study evaluation of robot assisted play for children with autism with a minimally expressive humanoid robot. In Proceedings of the 2nd International Conference on Advances in Computer-Human Interactions. IEEE, Los Alamitos, CA, 205-211. DOI : https:// doi.org/10.1109/ACHI.2009.32

[48] Morris Rosenberg. 1965. Society and the Adolescent Self-Image. Princeton University Press.

[49] Ori Rubin. 2015. Contact between parents and adult children: The role of time constraints, commuting and automobility. Journal of Transport Geography 49 (2015), 76-84. DOI : https://doi.org/10.1016/j.jtrangeo.2015.10.013

[50] Ryohei Sasama, Tomoharu Yamaguchi, and Keiji Yamada. 2011. An experiment for motivating elderly people with robot guided interaction. In Universal Access in Human-Computer Interaction: Users Diversity. Lecture Notes in Computer Science, Vol. 6766. Springer, Berlin, Germany, 214-223. DOI : https://doi.org/10.1007/978-3-642-21663-3_23

[51] Takanori Shibata and Kazuyoshi Wada. 2011. Robot therapy: A new approach for mental healthcare of the elderly-A mini-review. Gerontology 57 (2011), 378-386. DOI : https://doi.org/10.1159/000319015

[52] Masahiro Shiomi, Aya Nakata, Masayuki Kanbara, and Norihiro Hagita. 2017. A robot that encourages self-disclosure by hug. In Social Robotics. Lecture Notes in Computer Science, Vol. 10652. Springer, Cham, Switzerland, 324-333. DOI : https://doi.org/10.1007/978-3-319-70022-9_32

[53] Maki Suganuma. 1997. Self-disclosure and self-esteem in old age. Fapanese fournal of Educational Psychology 45, 4 (1997), 378-387 (in Japanese). DOI : https://doi.org/10.5926/jjep1953.45.4_378

[54] Yasir Tahir, Justin Dauwels, Daniel Thalmann, and Nadia Magnenat Thalmann. 2018. A user study of a humanoid robot as a social mediator for two-person conversations. International fournal of Social Robotics. Published online April 25, 2018. DOI : https://doi.org/10.1007/s12369-018-0478-3

[55] Adriana Tapus and Maja J. Matarić. 2008. Socially assistive robots: The link between personality, empathy, physiological signals, and task performance. In Proceedings of the AAAI Spring Symposium on Emotion, Personality, and Social Behavior. http://www.aaai.org/Library/Symposia/Spring/2008/ss08-04-021.php.

[56] Sabine Trepte and Michael Scharkow. 2016. Friends and lifesavers: How social capital and social support received in media environments contribute to well-being. In The Routledge Handbook of Media Use and Well-Being: International Perspectives on Theory and Research on Positive Media Effects, L. Reinecke and M. B. Oliver (Eds.). Routledge, 304-316. DOI : https://doi.org/10.4324/9781315714752

[57] Sherry Turkle. 2005. Relational artifacts/children/elders: The complexities of cybercompanions. In Proceedings of the CogSci 2005 Workshop: Toward Social Mechanisms of Android Science. 62-73.

[58] Takahisa Uchida, Hideyuki Takahashi, Midori Ban, Jiro Shimaya, Yuichiro Yoshikawa, and Hiroshi Ishiguro. 2017. A robot counseling system-What kinds of topics do we prefer to disclose to robots? In Proceedings of the 26th IEEE International Symposium on Robot and Human Interactive Communication. IEEE, Los Alamitos, CA, 207-212. DOI : https://doi.org/10.1109/ROMAN.2017.8172303

[59] Joseph B. Walther. 1996. Computer-mediated communication: Impersonal, interpersonal, and hyperpersonal interaction. Communication Research 23, 1 (1996), 3-43. DOI : https://doi.org/10.1177/009365096023001001

[60] Ran Zhao, Alexandros Papangelis, and Justine Cassell. 2014. Towards a dyadic computational model of rapport management for human-virtual agent interaction. In Intelligent Virtual Agents. Lecture Notes in Computer Science, Vol. 8627. Springer, Cham, Switzerland, 514-527. DOI : https://doi.org/10.1007/978-3-319-09767-1_62

Received April 2019; revised August 2019; accepted December 2019 